\title{
RB1 Polymorphism Contributes to the Efficacy of Platinum- Taxanes in Advanced Squamous Cell Lung Cancer
}

\author{
Di Liu ${ }^{1 \&}$, Wen Xu ${ }^{1 \&}$,Zhi-Wei Zhang ${ }^{1}$,Ji Qian ${ }^{4}$, Hui Zheng ${ }^{3}$, Jie Zhang ${ }^{2 *}$, Bo Su$^{1 *}$
}

\begin{abstract}
Background: RB1 (retinoblastoma 1) was reportedly one of the major determinative factors for sensitivity to taxanes in previous studies. In this study, we investigated the influence of RB1 single nucleotide polymorphisms (SNPs) on the efficacy of platinum-taxane regimens in advanced NSCLC patients. Materials and Methods: 234 cases of patients with advanced NSCLC who were treated with first-line platinum-taxane agents were enrolled in this study. Genomic DNA was extracted from patients' peripheral blood samples using a QIAamp DNA Maxi Kit, and genotyped by iSelect HD Bead-Chip. Results: Regression analyses were conducted through the univariate and multivariate Cox proportional hazards model in the 234 patients. The results showed that of the eight RB1 tagSNPs, only rs4151510 was a positive predictive factor for the advanced NSCLC patients treated with platinum taxanes regimen. The patients with $G / G$ genotype of $R B$ rs4151510 had longer overall survival (OS) than the non-G/G genotype $(p=0.018)$. The histology was also correlated with $O S$ in the whole advanced NSCLC patients. Three tagSNPs of RB1, rs4151510, rs4151465, rs9568036 were significantly associated with OS in the advanced NSCLC patients with squamous cell histology using Kaplan-Meier overall survival analysis stratified by histology. Conclusions: RB1 genomic variants were correlated with the efficacy of platinum-taxanes regimen. RB rs4151510 is an independent factor of the prognosis of NSCLC patients receiving platinum-taxane chemotherapy.
\end{abstract}

Keywords: Chemotherapy - RB1 - single nucleotide polymorphism - lung cancer

Asian Pac J Cancer Prev, 16 (2), 775-781

\section{Introduction}

Lung cancer is one of the leading causes of tumor related mortality in the world as well as in China (Chen et al., 2013). Non-small-cell lung cancer (NSCLC) accounts for approximately $80 \%$ and most patients are diagnosed in the advanced clinical stage (Ferlay et al., 2010). Nowadays, the treatment of platinum-based doublet chemotherapy remains the first-line option for NSCLC patients. Taxanes combined with platinum are commonly used in the first-line chemotherapy in many cancers, such as lung cancer or breast cancer (Rigas, 2004; De Laurentiis et al., 2008). Paclitaxel or docetaxel are microtubuletargeting anti-tumor drugs, which can bind to mitotic tubulin, and promote the microtubules assembly (Schiff et al., 1979), leading to mitotic arrest and, subsequently, cell apoptosis or death (Milross et al., 1996). Platinum-taxanes regimen had resulted in good response rates in advanced NSCLC patients (Fabi et al., 2004; Hirabayashi et al., 2004; Rozzi et al., 2010). However, because of the drugs resistant and adverse toxicity, such as nausea, vomiting, neutropenia, thrombocytopenia (Ozkaya et al., 2012), only less than $35 \%$ of patients display a positive response to platium-taxanes regimens, and the 5-year survival rate is less than $5 \%$ of unselected NSCLC patients.

$\mathrm{RB} 1$ is a tumor suppressor gene identified in the retinoblastoma, which is frequently inactivated in cancer and plays a pivotal role in the negative control of the cell cycle and in tumor progression. $\mathrm{Rb}$ protein is responsible for a major G1 checkpoint, blocking S-phase entry and cell growth by binding to the promoter of target genes with transcription factor E2F (Giacinti and Giordano, 2006). Functional loss of $\mathrm{Rb}$ results in deregulated activation of E2F to promote S-phase entry and apoptosis (Julian et al., 2008), and sensitizes cancers to taxol-induced cell death (Zhao et al., 2014). Several in vitro research also confirmed that the taxanes treatment can activate p38MAPK/ERK pathway (Suyama et al., 2004), or CDK/ $\mathrm{Rb} / \mathrm{E} 2 \mathrm{~F}$ pathway prior to induced apoptosis (Kurtyka et al., 2014). To investigate whether RB polymorphism can affect the efficacy of platinum-taxanes chemotherapy in clinical practice, we enrolled 234 advanced NSCLC patients treated with platinum-taxanes and attempted to illustrate the relationship between RB genotypes and the

${ }^{I}$ Central Laboratory, ${ }^{2}$ Department of oncology, ${ }^{3}$ Department of Thoracic Surgery, Shanghai Pulmonary Hospital, Tongji University School of Medicine, ${ }^{4}$ Fudan University Shanghai Cancer Center, State Key Laboratory of Genetic Engineering and MOE Key Laboratory of Contemporary Anthropology, School of Life Sciences, Fudan University, Shanghai, China \&Equal contributors *For correspondence: su_bo_s@hotmail.com, zhangjie2172@163.com 
chemotherapeutic efficacy of platinum-taxanes regimen in patients with advanced NSCLC.

\section{Materials and Methods}

\section{Patients}

234 patients diagnosed as clinical stage IIIA or IV NSCLC were enrolled in this study. All the patients received platinum-paclitaxel chemotherapy between March 2005 and January 2010, with no prior surgery, radiotherapy, or concurrent chemoradiotherapy. The regimens were as follows: either cisplatin $75 \mathrm{mg} / \mathrm{m}^{2}$ or carboplatin AUC 5 administered on day 1 every 3 weeks, in combination with paclitaxel $175 \mathrm{mg} / \mathrm{m}^{2}$ on day 1 every 3 weeks, or docetaxel $75 \mathrm{mg} / \mathrm{m}^{2}$ on day 1 every 3 weeks. The study was performed after approval of Medical Ethics Committee of the Shanghai Chest Hospital, Shanghai Zhongshan Hospital, Shanghai Changhai Hospital, and Shanghai Pulmonary Hospital with the patient's
A

C
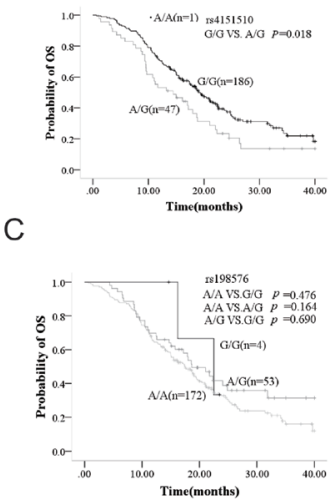

E

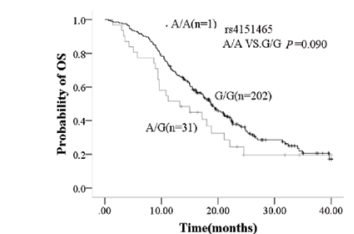

G

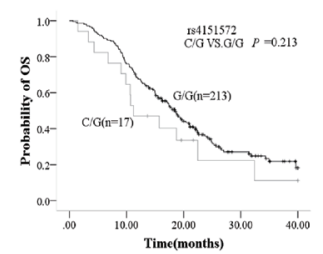

B

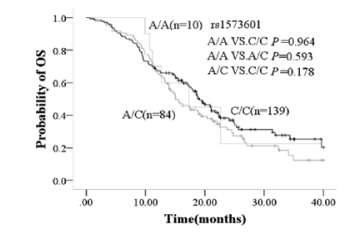

$\mathrm{D}$

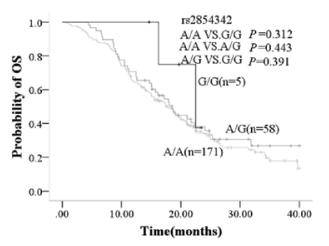

F

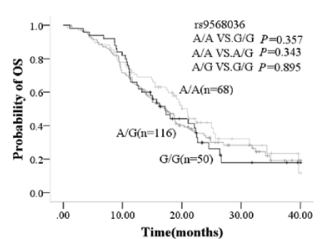

$\mathrm{H}$

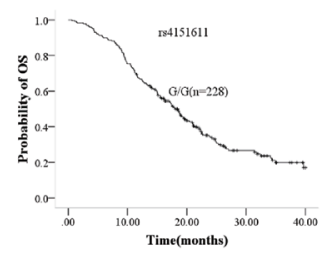

Figure 1. Kaplan-Meier Overall Survival Curves of whole Advanced NSCLC Patients Treated with Platinum-Taxanes Regimen. A) Kaplan-Meier curve of OS for rs4151510 in all patients with advanced NSCLC. B) Kaplan-Meier curve of OS for rs1573601 in all patients with advanced NSCLC. C) Kaplan-Meier curve of OS for rs198576 in all patients with advanced NSCLC. D) Kaplan-Meier curve of OS for rs2854342 in all patients with advanced NSCLC. E) Kaplan-Meier curve of OS for rs4151465 in all patients with advanced NSCLC. F) Kaplan-Meier curve of OS for rs9568036 in all patients with advanced NSCLC. G) Kaplan-Meier curve of OS for rs4151572 in all patients with advanced NSCLC. H) Kaplan-Meier curve of OS for rs4151611in all patients with advanced NSCLC information consents. All the patients had histologically confirmed NSCLC with the presence of at least one measurable and evaluable lesion. The cardiovascular, hepatic, hematologic, and renal functions of the patients have been examined to assess chemotherapy tolerance.

\section{RB1 tag SNPs selection}

The RB1 eight tag SNPs (rs1573601, rs198576, rs2854342, rs4151465, rs4151510, rs9568036, rs4151572 and rs4151611) were selected from the data of the HapMap SNP database (http://www.hapmap.org/). The SNPs were genotyped by iSelect HD Bead-Chip (Illumina, San Diego, CA, USA) with the following quality-control criteria: MAF $>0.05$, genotyping call rate $>0.95$; and GenCall score $>0.2$, correlation coefficient $\left(\mathrm{r}^{2}\right)$ threshold is 0.8 . Genomic DNA was extracted from patients' peripheral blood samples using the QIAamp DNA Maxi Kit (Qiagen GmbH, Hilden, Germany), following the manufacturer's instruction. The concordance among replicates was $>99.9 \%$. Analyzing the data and preparing reports were done using GeneMap software.

\section{Tumor response}

The overall survival (OS) was defined as the first day to receive chemotherapy treatment to the day of death
A

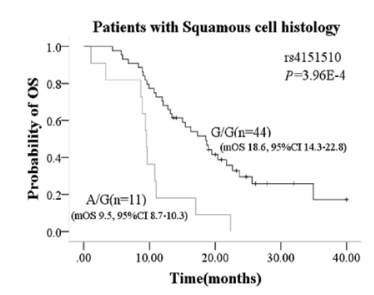

C

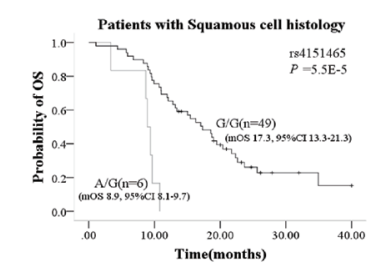

E

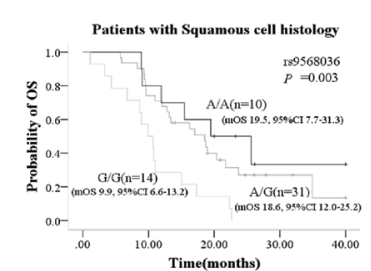

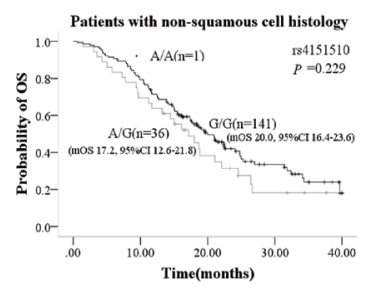

D

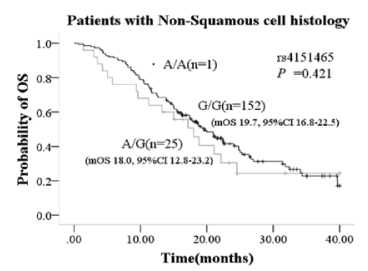

F

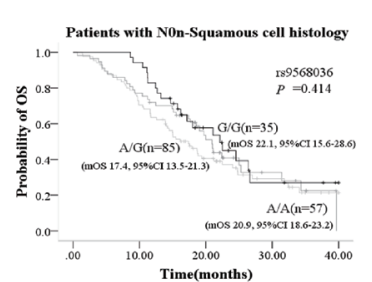

Figure 2. Kaplan-Meier Overall Survival Analysis of RB1 tagSNPs Genotype Stratified by Histology. A) Kaplan-Meier curve of OS for rs4151510 in the patients of squamous cell histology sub-group. B) Kaplan-Meier curve of OS for rs4151510 in the patients of non-squamous cell histology sub-group C) Kaplan-Meier curve of OS for rs4151465 in the patients of squamous cell histology sub-group. D) Kaplan-Meier curve of OS for rs4151465 in the patients of non-squamous cell histology sub-group E) Kaplan-Meier curve of OS for rs9568036 in the patients of squamous cell histology sub-group. F) KaplanMeier curve of OS for rs9568036 in the patients of non-squamous cell histology sub-group 
RB1 Polymorphism Contributes to the Efficacy of Platinum-Taxanes in Advanced Squamous Cell Lung Cancer

or to the latest follow-up according to the Response Evaluation Criteria in Solid Tumors (RECIST). The shortterm responses to chemotherapy treatment were evaluated after the first 2 cycles. Complete response (CR), partial response (RR) and sTable disease (SD) were known as the disease control rate (DCR). The objective response rate

Table 1. Association between RB1 Tag SNPs and OS in NSCLC Patients

\begin{tabular}{|c|c|c|c|c|c|}
\hline Genotype & & $\mathrm{N}(\%)$ & $\mathrm{mOS}(95 \% \mathrm{CI}), \mathrm{mo}$ & HR (95\% CI) & $P$-value \\
\hline rs1573601 & & & & & 0.423 \\
\hline $\mathrm{C} / \mathrm{C}$ & 139 & $(59.7 \%)$ & $18.8(16.4-21.3)$ & 1 & $\mathrm{R}$ \\
\hline $\mathrm{A} / \mathrm{C}$ & 84 & $(36.1 \%)$ & $15.0(11.8-18.3)$ & $1.2(0.9-1.7)$ & 0.195 (CC vs.AC) \\
\hline $\mathrm{A} / \mathrm{A}$ & 10 & $(4.3 \%)$ & $17.3(6.8-27.8)$ & $1.0(0.4-2.3)$ & 0.994 (CC vs.AA) \\
\hline rs 198576 & & & & & 0.308 \\
\hline $\mathrm{A} / \mathrm{A}$ & 172 & $(75.1 \%)$ & $17.4(14.6-20.2)$ & 1 & $\mathrm{R}$ \\
\hline $\mathrm{A} / \mathrm{G}$ & 53 & $(23.1 \%)$ & $18.7(14.1-23.3)$ & $0.8(0.5-1.1)$ & 0.163 (AG vs.AA) \\
\hline $\mathrm{G} / \mathrm{G}$ & 4 & $(1.7 \%)$ & $22.5(12.4-32.6)$ & $0.6(0.1-2.4)$ & 0.47 (AA vs.GG) \\
\hline rs2854342 & & & & & 0.467 \\
\hline $\mathrm{A} / \mathrm{A}$ & 171 & $(73.1 \%)$ & $17.7(14.6-20.7)$ & 1 & $\mathrm{R}$ \\
\hline $\mathrm{A} / \mathrm{G}$ & 58 & $(24.8 \%)$ & $18.6(15.3-21.9)$ & $0.9(0.6-1.2)$ & 0.44 (AG vs.AA) \\
\hline $\mathrm{G} / \mathrm{G}$ & 5 & $(2.1 \%)$ & $22.5(13.1-31.9)$ & $0.5(0.1-2.0)$ & 0.313 (GG vs.AA) \\
\hline rs4151465 & & & & & 0.144 \\
\hline $\mathrm{G} / \mathrm{G}$ & 202 & $(86.3 \%)$ & $18.7(16.4-21.1)$ & 1 & $\mathrm{R}$ \\
\hline $\mathrm{A} / \mathrm{G}$ & 31 & $(13.2 \%)$ & $13.2(6-20.4)$ & $1.5(0.9-2.3)$ & 0.092 (AG vs.GG) \\
\hline $\mathrm{A} / \mathrm{A}$ & 1 & $(0.4 \%)$ & 11.4 & $2.9(0.4-21.3)$ & 0.283 (AA vs.GG) \\
\hline rs4151510 & & & & & $0.038^{*}$ \\
\hline $\mathrm{G} / \mathrm{G}$ & 186 & $(79.5 \%)$ & $19.4(17.0-21.8)$ & 1 & $\mathrm{R}$ \\
\hline $\mathrm{A} / \mathrm{G}$ & 47 & $(20.1 \%)$ & $14.5(8.1-20.9)$ & $1.6(1.1-2.3)$ & $0.018 *(\mathrm{AG}$ vs.GG) \\
\hline $\mathrm{A} / \mathrm{A}$ & 1 & $(0.4 \%)$ & 11.4 & $3.1(0.4-22.2)$ & 0.264 (AA vs.GG) \\
\hline rs9568036 & & & & & 0.569 \\
\hline $\mathrm{A} / \mathrm{G}$ & 116 & $(49.6 \%)$ & $17.4(14.4-20.4)$ & 1 & $\mathrm{R}$ \\
\hline $\mathrm{A} / \mathrm{A}$ & 68 & $(29.1 \%)$ & $20.9(18.8-23.1)$ & $0.8(0.6-1.2)$ & 0.344 (AA vs.AG) \\
\hline $\mathrm{G} / \mathrm{G}$ & 50 & $(21.4 \%)$ & $17.2(13.6-20.7)$ & $1.0(0.7-1.5)$ & 0.881 (GG vs.AG) \\
\hline rs4151572 & & & & & 0.216 \\
\hline $\mathrm{G} / \mathrm{G}$ & 213 & $(92.6 \%)$ & $18.6(16.4-20.7)$ & 1 & $\mathrm{R}$ \\
\hline $\mathrm{C} / \mathrm{G}$ & 17 & $(7.4 \%)$ & $11.2(4.7-17.7)$ & $1.4(0.8-2.5)$ & 0.216 (CG vs. GG) \\
\hline \multicolumn{6}{|l|}{ rs4151611 } \\
\hline $\mathrm{G} / \mathrm{G}$ & 228 & $(100 \%)$ & $18.0(16.0-20.0)$ & NA & NA \\
\hline
\end{tabular}

Table 3. Association between RB1 tagSNPs and OS in Patients with Squamous or Non-Squamous Lung Cancer

\begin{tabular}{|c|c|c|c|c|c|c|c|c|}
\hline \multirow{2}{*}{$\begin{array}{l}\text { SNP ID } \\
\text { genotype }\end{array}$} & \multicolumn{4}{|c|}{ Squamous cell patients } & \multicolumn{4}{|c|}{ Non-squamous cell patients } \\
\hline & $\mathrm{e} N(\%)$ & $\mathrm{mOS}(95 \% \mathrm{CI}), \mathrm{mo}$ & $\mathrm{HR}(95 \% \mathrm{CI})$ & $P$-value & $\mathrm{N}(\%) \mathrm{m}$ & $\mathrm{mOS}(95 \% \mathrm{CI}), \mathrm{mo}$ & $\mathrm{HR}(95 \% \mathrm{CI})$ & $P$-value \\
\hline \multicolumn{9}{|l|}{ rs1573601 } \\
\hline $\mathrm{C} / \mathrm{C}$ & $26(47.3 \%)$ & $17.0(7.6-26.4)$ & 1 & 0.443(CC vs.AA) & $112(63.3 \%)$ & $19.1(16.4-21.8)$ & 1 & 0.406 (CC vs. AA) \\
\hline $\mathrm{A} / \mathrm{C}$ & $25(45.5 \%)$ & $13.4(10.0-16.8)$ & $1.5(0.8-2.8)$ & 0.247 (AC vs.CC) & $59(33.3 \%)$ & $15.7(10.8-20.6)$ & $1.2(0.8-1.7)$ & 0.464(AC vs.CC) \\
\hline $\mathrm{A} / \mathrm{A}$ & $4(7.3 \%)$ & $10.7(3.5-17.9)$ & $1.7(0.6-5.1)$ & 0.652(AA vs.AC) & $6(3.4 \%)$ & 0 & $0.5(0.1-2.2)$ & 0.229 (AA vs.AC) \\
\hline \multicolumn{9}{|l|}{ rs198576 } \\
\hline $\mathrm{A} / \mathrm{A}$ & $40(74.1 \%)$ & $16.3(10.7-21.8)$ & 1 & NA(GG vs. AA) & $131(75.3 \%)$ & $18.6(15.2-21.9)$ & 1 & 0.514 (GG vs. AA) \\
\hline $\mathrm{A} / \mathrm{G}$ & $14(25.9 \%)$ & $11.0(1.0-21.0)$ & $1.1(0.6-2.3)$ & 0.727(AG vs.AA) & $39(22.4 \%)$ & $24.0(16.3-31.8)$ & $0.7(0.4-1.1)$ & 0.088(AG vs.AA) \\
\hline $\mathrm{G} / \mathrm{G}$ & 0 & & & NA(GG vs.AG) & $4(2.3 \%)$ & $22.5(12.4-35.6)$ & $0.6(0.2-2.6)$ & 0.943 (GG vs.AG) \\
\hline \multicolumn{9}{|c|}{ rs2854342 } \\
\hline $\mathrm{A} / \mathrm{A}$ & $41(74.5 \%)$ & $16.3(11.4-21.1)$ & 1 & NA(GG vs. AA) & $129(72.5 \%)$ & $18.7(14.3-23.1)$ & 1 & 0.364 (GG vs. AA) \\
\hline $\mathrm{A} / \mathrm{G}$ & $14(25.5 \%)$ & ) $11.0(1.0-21.0)$ & $1.1(0.5-2.2)$ & 0.764(AG vs.AA) & $44(24.7 \%)$ & $19.1(14.6-23.5)$ & $0.8(0.5-1.3)$ & 0.368(AG vs.AA) \\
\hline $\mathrm{G} / \mathrm{G}$ & 0 & NA & NA & NA(GG vs.AG) & $5(2.8 \%)$ & $22.5(13.1-31.9)$ & $0.5(0.1-2.1)$ & $0.527(\mathrm{GG}$ vs.AG) \\
\hline \multicolumn{9}{|c|}{ rs4151465 } \\
\hline $\mathrm{G} / \mathrm{G}$ & $49(89.1 \%)$ & $17.3(13.3-21.3)$ & 1 & $5.5 \mathrm{E}-5^{*}(\mathrm{GG}$ vs.AG $)$ & $152(85.4 \%)$ & $19.7(16.8-22.5)$ & 1 & 0.421 (GG vs.AG) \\
\hline $\mathrm{A} / \mathrm{G}$ & $6(10.9 \%)$ & $8.9(8.1-9.7)$ & $6.0(2.2-16.2)$ & NA(AG vs.AA) & $25(14.0 \%)$ & $18.0(12.8-23.2)$ & $1.2(0.7-2.1)$ & 0.433(AG vs.AA) \\
\hline $\mathrm{A} / \mathrm{A}$ & 0 & NA & NA & NA(GG vs. AA) & $1(0.6 \%)$ & 11.4 & $3.3(0.4-23.7)$ & 0.201 (GG vs. AA) \\
\hline \multicolumn{9}{|c|}{ rs4151510 } \\
\hline $\mathrm{G} / \mathrm{G}$ & $44(80 \%)$ & $18.6(14.3-22.8)$ & 1 & $3.96 \mathrm{E}-4 *(\mathrm{GG}$ vs. AG & J) $141(79.2 \%)$ & $20.0(16.4-23.6)$ & 1 & 0.221 (GG vs. AG) \\
\hline $\mathrm{A} / \mathrm{G}$ & $11(20 \%)$ & $9.5(8.7-10.3)$ & $3.4(1.7-7.0)$ & NA(AG vs.AA) & $36(20.2 \%)$ & $17.2(12.6-21.8)$ & $1.3(0.8-2.0)$ & 0.371(AG vs.AA) \\
\hline $\mathrm{A} / \mathrm{A}$ & 0 & NA & NA & $\mathrm{NA}(\mathrm{GG}$ vs.AA) & $1(0.6 \%)$ & 11.4 & $3.4(0.5-24.4)$ & 0.199 (GG vs.AA) \\
\hline \multicolumn{9}{|c|}{ rs9568036 } \\
\hline $\mathrm{A} / \mathrm{G}$ & $31(56.4 \%)$ & $18.6(12.0-25.2)$ & 1 & $0.004 *(\mathrm{GG}$ vs. $\mathrm{AG})$ & $85(48.0 \%)$ & $17.4(13.5-21.3)$ & 1 & 0.238 (GG vs. AG) \\
\hline $\mathrm{A} / \mathrm{A}$ & $10(18.2 \%)$ & $19.5(7.7-31.3)$ & $0.7(0.3-1.7)$ & 0.447(AG vs.AA) & $57(32.2 \%)$ & $20.9(18.6-23.2)$ & $0.9(0.6-1.3)$ & 0.463 (AG vs.AA) \\
\hline $\mathrm{G} / \mathrm{G}$ & $14(25.5 \%)$ & $9.9(6.6-13.2)$ & $2.7(1.4-5.4)$ & $0.004 *(\mathrm{GG}$ vs.AA $)$ & $35(19.8 \%)$ & $22.1(15.6-28.6)$ & $0.7(0.5-1.2)$ & 0.591 (GG vs.AA) \\
\hline \multicolumn{9}{|c|}{ rs4151572 } \\
\hline G/G & $50(90.9 \%)$ & $15.5(10.8-20.1)$ & 1 & $\mathrm{R}$ & $162(93.1 \%)$ & $18.8(16.2-21.5)$ & 1 & $\mathrm{R}$ \\
\hline $\mathrm{C} / \mathrm{G}$ & $5(9.1 \%)$ & $10.7(2.3-19.1)$ & $2.0(0.7-5.8)$ & $0.174(\mathrm{GG}$ vs.CG) & $12(6.9 \%)$ & $11.2(2.5-19.9)$ & $1.3(0.7-2.5)$ & 0.468 (GG vs.CG) \\
\hline
\end{tabular}


Table 2. Multivariate Cox's Regression Analysis in the whole Patients for Overall Survival Prognostic Factor

\begin{tabular}{|c|c|c|}
\hline Variables & $\mathrm{HR}(95 \% \mathrm{CI})$ & $\mathrm{P}$ \\
\hline Median age $(<60 v s .>60)$ & $1.1(0.8-1.5)$ & 0.722 \\
\hline $\operatorname{Sex}(\mathrm{F}$ vs. M) & $1.0(0.6-2.6)$ & 0.98 \\
\hline Smoking status(Smoker vs. Never) & $1.2(0.8-1.7)$ & 0.301 \\
\hline Histology & & $0.048 *$ \\
\hline Adenocarcinoma & 1 & $\mathrm{R}$ \\
\hline Squamous & $1.4(0.9-2.0)$ & 0.114 \\
\hline Adenosquamous & $8.9(1.2-66.1)$ & 0.032 \\
\hline Others\# & $0.8(0.4-1.5)$ & 0.432 \\
\hline Clinical Stage(III $v s$ IV): & $1.3(0.9-1.9)$ & 0.151 \\
\hline rs 1573601 & & 0.772 \\
\hline $\mathrm{C} / \mathrm{C}$ & 1 & $\mathrm{R}$ \\
\hline $\mathrm{A} / \mathrm{C}$ & $1.0(0.5-2.0)$ & 0.909 \\
\hline $\mathrm{A} / \mathrm{A}$ & $0.7(0.2-1.7)$ & 0.569 \\
\hline rs198576 & & 0.118 \\
\hline $\mathrm{A} / \mathrm{A}$ & 1 & $\mathrm{R}$ \\
\hline $\mathrm{A} / \mathrm{G}$ & $0.8(0.5-1.1)$ & 0.183 \\
\hline $\mathrm{G} / \mathrm{G}$ & $0.8(0.2-3.2)$ & 0.738 \\
\hline rs2854342 & & 0.438 \\
\hline $\mathrm{A} / \mathrm{A}$ & 1 & $\mathrm{R}$ \\
\hline $\mathrm{A} / \mathrm{G}$ & $1.8(0.7-4.7)$ & 0.199 \\
\hline $\mathrm{G} / \mathrm{G}$ & ND & NA \\
\hline rs4151465 & & 0.083 \\
\hline $\mathrm{G} / \mathrm{G}$ & 1 & $\mathrm{R}$ \\
\hline $\mathrm{A} / \mathrm{G}$ & $1.6(1.0-2.5)$ & 0.049 \\
\hline $\mathrm{A} / \mathrm{A}$ & $3.2(0.4-22.8)$ & 0.256 \\
\hline rs4151510 & & $0.035 *$ \\
\hline $\mathrm{G} / \mathrm{G}$ & 1 & $\mathrm{R}$ \\
\hline $\mathrm{A} / \mathrm{G}$ & $1.6(1.1-2.3)$ & $0.016^{*}$ \\
\hline $\mathrm{A} / \mathrm{A}$ & $3.1(0.4-22.1)$ & 0.27 \\
\hline rs9568036 & & 0.38 \\
\hline $\mathrm{A} / \mathrm{G}$ & 1 & $\mathrm{R}$ \\
\hline $\mathrm{A} / \mathrm{A}$ & $0.8(0.5-1.2)$ & 0.231 \\
\hline $\mathrm{G} / \mathrm{G}$ & $1.0(0.7-1.6)$ & 0.916 \\
\hline \multicolumn{3}{|l|}{ rs4151572 } \\
\hline $\mathrm{G} / \mathrm{G}$ & 1 & $\mathrm{R}$ \\
\hline $\mathrm{C} / \mathrm{G}$ & $1.5(0.8-2.6)$ & 0.196 \\
\hline
\end{tabular}

$* p<0.05$; \# : Other histology included mixed-cell, neuroendocrine carcinoma, or undifferentiated carcinoma; Abbreviation: ND, no data; NA, not available; R, reference

(ORR) consisted of complete response (CR) and partial response $(\mathrm{PR})$.

\section{Statistical analysis}

All statistical analyses were finished using SPSS $®$ version 20.0 (SPSS Inc., Chicago, IL, USA). The association of OS for the NSCLC patients with RB tagSNPs genotypes was evaluated by the KaplanMeier curves verified by log-rank tests. Univariate and multivariate analyses were executed utilizing the Cox proportional hazard model to validate the significant factors related to the OS. All statistical analyses were two-sided, and differences were regarded as statistically significant at $p<0.05$.

\section{Results}

Patients' clinical characteristics and RB1 tagSNPS genotype

The clinical characteristics of the total 234 patients were shown in Table S1. The cohort consisted of 174
Table 3. Clinical Characteristics of the Recruited Patients with Advanced NSCLC

\begin{tabular}{lrrc}
\hline Variables & Total $(\mathrm{N})=234$ & mOS $(95 \% \mathrm{CI})$ & $\mathrm{P}$ \\
\hline $\begin{array}{l}\text { Median age }(\mathrm{y}) \\
<60\end{array}$ & 58.74 & & \\
$\geq 60$ & $106(45.3 \%)$ & $18.7(14.5-22.9)$ & 0.534 \\
Sex: & $128(54.7 \%)$ & $17.4(14.7-20.1)$ & \\
$\quad$ Male & $174(74.4 \%)$ & $17.2(14.8-19.6)$ & 0.146 \\
Female & $60(25.6 \%)$ & $21.0(17.2-24.8)$ & \\
Smoking status: & & & \\
$\quad$ Non-smoker & $88(37.6 \%)$ & $19.7(17.4-22.0)$ & 0.175 \\
Smoker & $146(62.4 \%)$ & $17.3(14.4-20.2)$ & \\
Histology & & & \\
$\quad$ Adenocarcinoma & $158(67.8 \%)$ & $18.7(15.9-21.5)$ & 0.307 \\
Squamous & $55(23.6 \%)$ & $15.5(10.6-20.4)$ & 0.132 \\
Adenosquamous & $3(1.3 \%)$ & $21.2(0-43.6)$ & 0.685 \\
Others & $17(7.3 \%)$ & $24.8(20.7-29.0)$ & 0.385 \\
Clinical Stage: & & & 0.479 \\
IIIa & $16(6.8 \%)$ & $18.6(5.7-31.5)$ & \\
IIIb & $61(26.1 \%)$ & $19.0(15.8-22.1)$ & \\
IV & $157(67.1 \%)$ & $17.5(14.3-20.8)$ & \\
\hline
\end{tabular}

ather histology included mixed-cell, neuroendocrine carcinoma, or undifferentiated carcinoma; ECOG PS: Eastern Cooperative Oncology Group performance status; * $p<0.05$

males and 60 females with the median age of 58 years, ranged from 26 to 80 years old. 146 patients were smokers, 88 patients were never-smoker. All the patients were diagnosed at IIIa/IIIb $(32.9 \%)$ or IV clinical stage (67.1\%) with the Eastern Cooperative Oncology Group performance status (ECOG PS) 1. The histology of NSCLC included adenocarcinoma (67.6\%), squamous cell carcinoma $(23.8 \%)$, adenosquamous cell carcinoma (1.6\%) or other histological types, such as mixed-cell, neuroendocrine carcinoma, or undifferentiated carcinoma. The association of clinical characteristics with OS was analyzed using Kaplan-Meier curve test, No significantly association was observed between the OS and age $(<=60$ or $>60$ ), sex (M or F), smoking status (ever or never), histology (AD, SQ, Ad-AQ or other types), or clinical stage (IIIa/IIIb or IV clinical stage). (Table S1).

The genotype frequencies and the minor allele frequencies (MAF) of eight RB tagSNPs were listed in Table S2. No significant difference was observed between the MAFs of our current data and the CHB data from the HapMap SNP database 3. However, the MAF in CEU population was significantly different to that of the current data or CHB population in HapMap $(p<0.05)$.

RB1 rs4151510 genotype is associated with the overall survival of NSCLC patients treated with platinumpaclitaxel agents

The association of RB1 tagSNPs genotypes with the OS of NSCLC patients treated with platinumtaxanes regimens was analyzed using univariate Cox's proportional hazards regression analysis (Table 1). Among the 8 RB1 tagSNPs, rs4151510 was significantly correlated with OS in the advanced NSCLC patients $(\mathrm{P}=0.038)$. The patients with $\mathrm{G} / \mathrm{G}$ genotype (mOS 19.4, 95\%CI 17.0-21.8) of rs4151510 had longer survival than $\mathrm{A} / \mathrm{G}$ genotype $(\operatorname{mOS} 14.5,95 \% \mathrm{CI} 8.1-20.9, p=0.018$ ) 
RB1 Polymorphism Contributes to the Efficacy of Platinum-Taxanes in Advanced Squamous Cell Lung Cancer

Table 4. RB1 Eight Tag SNPs MAF and Genotype Frequencies of Current Data, CHB, and CEU Data from HapMap SNP Database 3.

\begin{tabular}{|c|c|c|c|c|c|}
\hline \multirow[t]{2}{*}{ RB1 tagSNP } & \multirow[t]{2}{*}{ Location } & \multicolumn{4}{|c|}{ Allel frequencies } \\
\hline & & Current Data & CHB & CEU & $p$ \\
\hline rs1573601 & 5 'near gene & & & & \\
\hline MAF & & $0.223(\mathrm{~A})$ & $0.22(\mathrm{~A})$ & $0.230(\mathrm{~A})$ & 0.95 \\
\hline $\mathrm{A} / \mathrm{A}$ & & $10 \quad(4.3 \%)$ & $2(2.4 \%)$ & $14 \quad(6.2 \%)$ & \\
\hline $\mathrm{A} / \mathrm{C}$ & & $84(36.1 \%)$ & $32(39 \%)$ & $76(33.6 \%)$ & \\
\hline $\mathrm{C} / \mathrm{C}$ & & $139(59.7 \%)$ & $48(58.5 \%)$ & $136(60.2 \%)$ & \\
\hline rs198576 & intron & & & & \\
\hline MAF & & $0.133(\mathrm{G})$ & $0.100(\mathrm{G})$ & $0.025(\mathrm{G})$ & $0.007 *$ \\
\hline $\mathrm{A} / \mathrm{A}$ & & $172(75.1 \%)$ & $76(84.4 \%)$ & $114(95 \%)$ & \\
\hline $\mathrm{A} / \mathrm{G}$ & & $53(23.1 \%)$ & $10(11.1 \%)$ & 0 & \\
\hline $\mathrm{G} / \mathrm{G}$ & & $4 \quad(1.7 \%)$ & $4(4.4 \%)$ & $6 \quad(0.5 \%)$ & \\
\hline rs2854342 & intron & & & & \\
\hline MAF & & $0.145(\mathrm{G})$ & $0.122(\mathrm{G})$ & $0.027(\mathrm{G})$ & 0 \\
\hline $\mathrm{A} / \mathrm{A}$ & & $171(73.1 \%)$ & $62(75.6 \%)$ & $214(94.7 \%)$ & \\
\hline $\mathrm{A} / \mathrm{G}$ & & $58(24.8 \%)$ & $20(24.4 \%)$ & $12 \quad(5.3 \%)$ & \\
\hline $\mathrm{G} / \mathrm{G}$ & & $5 \quad(2.1 \%)$ & 0 & 0 & \\
\hline rs4151465 & intron & & & & \\
\hline MAF & & $0.071(\mathrm{~A})$ & $0.056(\mathrm{~A})$ & $0(\mathrm{~A})$ & $1.10 \mathrm{E}-05^{*}$ \\
\hline $\mathrm{A} / \mathrm{A}$ & & $1 \quad(0.4 \%)$ & 0 & 0 & \\
\hline $\mathrm{A} / \mathrm{G}$ & & $31(13.2 \%)$ & $10(11.1 \%)$ & 0 & \\
\hline $\mathrm{G} / \mathrm{G}$ & & $202(86.3 \%)$ & $80(88.9 \%)$ & $160(100 \%)$ & \\
\hline rs4151510 & intron & & & & \\
\hline MAF & & $0.105(\mathrm{~A})$ & $0.073(\mathrm{~A})$ & $0.119(\mathrm{~A})$ & 0.255 \\
\hline $\mathrm{A} / \mathrm{A}$ & & $1 \quad(0.4 \%)$ & 0 & 0 & \\
\hline $\mathrm{A} / \mathrm{G}$ & & $47(20.1 \%)$ & $12(14.6 \%)$ & $54(23.9 \%)$ & \\
\hline $\mathrm{G} / \mathrm{G}$ & & $186(79.5 \%)$ & $70(85.4 \%)$ & $172(11.9 \%)$ & \\
\hline rs9568036 & intron & & & & \\
\hline MAF & & $0.462(\mathrm{G})$ & $0.402(\mathrm{G})$ & $0.376(\mathrm{G})$ & $0.029^{*}$ \\
\hline $\mathrm{A} / \mathrm{A}$ & & $68 \quad(29.1 \%)$ & $32(39 \%)$ & $90(39.8 \%)$ & \\
\hline $\mathrm{A} / \mathrm{G}$ & & $116(49.6 \%)$ & $34(41.5 \%)$ & $102(45.1 \%)$ & \\
\hline $\mathrm{G} / \mathrm{G}$ & & $50(21.4 \%)$ & $16(19.5 \%)$ & $34(15 \%)$ & \\
\hline rs4151572 & intron & & & & \\
\hline MAF & & $0.037(\mathrm{C})$ & $0.067(\mathrm{C})$ & 0 & $0.001 *$ \\
\hline $\mathrm{C} / \mathrm{C}$ & & 0 & 0 & 0 & \\
\hline $\mathrm{C} / \mathrm{G}$ & & $17 \quad(7.4 \%)$ & $12(13.3 \%)$ & 0 & \\
\hline $\mathrm{G} / \mathrm{G}$ & & $213(92.6 \%)$ & $78(86.7 \%)$ & $120(100 \%)$ & \\
\hline rs4151611 & intron & & & & \\
\hline MAF & & 0 & $0.256(\mathrm{~A})$ & $0.033(\mathrm{~A})$ & 0 \\
\hline $\mathrm{A} / \mathrm{A}$ & & 0 & $10(11.1 \%)$ & 0 & \\
\hline $\mathrm{A} / \mathrm{G}$ & & 0 & $26(28.9 \%)$ & $8 \quad(6.7 \%)$ & \\
\hline $\mathrm{G} / \mathrm{G}$ & & $228(100 \%)$ & $54(60 \%)$ & $112(93.3 \%)$ & \\
\hline
\end{tabular}

(Table 1, Figure 1). It suggested that RB rs4151510 G/G genotype was a favorable factor for the survival of the advanced NSCLC patients who received the platinumpaclitaxel regimen.

The multivariate Cox's proportional hazards regression analysis suggested that age, sex, smoking status, or clinical stage (tumor-node-metastasis stage), etc. were not independent predictive factors except histology. The results showed that histology was significantly correlation with OS in this cohort study $(p=0.048)$. Among the 8 RB1 tagSNPs, only RB rs4151510 was associated with the overall survival of the patients $(p=0.035)$ (Table 3$)$, which suggested that RB rs4151510 was an independent risk factor for the OS and contributed to the efficacy of platinum-taxanes regimen in multivariate analysis (Table 2).

RB1 rs4151510 is predominately associated with OS in the patients with squamous cell histology who were treated with platinum-paclitaxel agents.

We also investigated the influence of RB rs4151510 genotype on different histology using Kaplan-Meier overall survival analysis, and found that RB rs4151510 genotype predominantly contributed towards the efficacy of platinum-paclitaxel regimen in the patients with squamous carcinoma ( $p=3.96 \mathrm{E}-4)$; but was not associated with non-squamous histological types $(p=0.229)$ (Figure 2). In patients with squamous carcinoma, $\mathrm{G} / \mathrm{G}$ genotype (mOS 18.6, 95\%CI 14.3-22.8) has longer OS than A/G genotype (mOS 9.5, 95\%CI 8.7-10.3) ( $p=3.96 \mathrm{E}-4)$. Therefore, RB1 rs4151510 G/G genotype was an independent protective factor in patients with advanced squamous cell carcinoma treated with platinum-paclitaxel regimens. Besides, RB1 rs4151465 and rs9868036 were significantly association with OS in the squamous cell histology advanced NSCLC patients (Table 3) 


\section{Discussion}

Platinum-based combination chemotherapy is currently considered to be a standard treatment for advanced NSCLC. Taxanes combined with platinum was one of the most common regimens in the firstline treatment of NSCLC. Taxanes target the tubulin/ microtubule system, stabilize microtubules and promote microtubule assembly, thereby inhibit the process of cell division (Manfredi et al., 1982; Wang et al., 2000). Taxanes can activate several signaling pathways to induce cell cycle arrest or apoptosis, such as, the c-Jun N-terminal kinase/stress-activated protein kinase (JNK/SAPK) (Lee et al., 1998), or ERK and subsequent RB phosphorylation (Suyama et al., 2004).RB protein was previously reported as a determinant of the sensitivity to taxanes in several in vitro studies (Suyama et al., 2004),

The dephosphorylated $\mathrm{Rb}$ protein confers the higher sensitivity to chemotherapy drugs, and the resistance of chemotherapy was correlated with the phosphorylated $\mathrm{Rb}$ protein (Yamamoto et al., 1998). So far, whether the polymorphism of RB1 gene is associated with the efficacy of Taxanes has not been investigated. In this study, we reported that RB rs4151510 was an independent prognostic factor for OS in the advanced NSCLC patients who were treated with platinum-taxanes regimen through univariate and multivariate Cox's regression analysis. The NSCLC with G/G genotype of RB1 rs4151510 was more sensitive than with A/A genotype or A/G genotype when treated with platinum-taxanes agents. Though this RB1 SNP can not be regarded as a site with comparably strong effect to predict clinical outcomes ( $p=0.03$ for all the NSCLC, $p=3.96 \mathrm{E}-4$ for squamous cell Lung cancer), this finding provides a corroborative evidence for the involvement of RB1 in the sensitivity of platinum-taxanes in NSCLC patients, especially in squamous cell lung cancer.

It was reported that that $\mathrm{RB}$ was associated with the squamous cell carcinomas ( $\mathrm{SCC}$ ) of nonmelanoma skin cancer and the head and neck and was considered as a biomarker for the chemotherapy outcome (Martinez-Cruz et al., 2008; van den Broek et al., 2009). In esophageal squamous cell carcinoma, inactive pRB was significant associated with with adverse prognosis (Contu et al., 2007; Wang et al., 2013; Dey et al., 2014) . Our results present that RB1 polymorphism was predominately associated with the efficacy of platinum-taxanes in squamous cell lung cancer. In the squamous cell histology sub-group, the overall survival of patients with $\mathrm{G} / \mathrm{G}$ genotype of rs4151510 was longer than the A/A or A/G genotype patients ( $p=3.96, \mathrm{E}-4)$; but no significance was observed for the non-squamous cell histology sub-group $(p=0.229)$. The diverse results of histological types may be due to the more frequent RB1 dysfunction in squamous cell cancer, which is in agreement with the former researches.

In conclusion, we investigated the relationship of between RB1 polymorphism and the clinical outcome of platinum-taxanes in NSCLC patients. The data suggested that RB1 rs4151510 was associated with the efficacy of platinum-taxanes regiments especially in squamous cell lung cancer. It must be pointed out, however, that these observations need to be substantiated with additional follow-up studies with a larger number of individuals with NSCLC. The mechanism of RB rs4151510 affecting the efficacy of platinum-taxanes chemotherapy also needs to be further studied.

\section{Acknowledgements}

This work was supported by the Science and Technology Commission of Shanghai Municipality (No. 12NM0500800, No. 12ZR1426100, No.09411960500,) and Shanghai Health Bureau Foundation (No. 201440397).

\section{References}

Chen W, Zheng R, Zhang S, et al (2013). Report of incidence and mortality in China cancer registries, 2009. Chin J Cancer Res, 25, 10-21.

Contu SS, Contu PC, Damin DC, et al (2007). pRB expression in esophageal mucosa of individuals at high risk for squamous cell carcinoma of the esophagus. World J Gastroenterol, 13, 1728.

De Laurentiis M, Cancello G, D’Agostino D, et al (2008). Taxane-based combinations as adjuvant chemotherapy of early breast cancer: A meta-analysis of randomized trials. $J$ Clinical Oncol, 26, 44-53.

Dey B, Raphael V, Khonglah Y, et al (2014). Immunohistochemical Analysis of P53 and PRB in Esophageal Squamous Cell Carcinoma. J Clin Diagn Res, 8, 1-3.

Fabi A, Barduagni M, Ferraresi V, et al (2004). The combination of carboplatin and weekly paclitaxel: a safe and active regimen in advanced non small-cell lung cancer patients. A phase I-II study. J Exp Clin Cancer Res, 23, 25-32.

Ferlay J, Shin HR, Bray F, et al (2010). Estimates of worldwide burden of cancer in 2008: GLOBOCAN 2008. Int J Cancer, 127, 2893-917.

Giacinti C, Giordano A (2006). RB and cell cycle progression. Oncogene, 25, 5220-7.

Hirabayashi M, Endoh K, Teramachi M, et al (2004). Phase II study of carboplatin and weekly paclitaxel combination chemotherapy in advanced non-small cell lung cancer: a Kansai Clinical Oncology Group study. Lung Cancer, 44, 355-62.

Julian LM, Palander O, Seifried LA, et al (2008). Characterization of an E2F1-specific binding domain in pRB and its implications for apoptotic regulation. Oncogene, 27, 1572-9.

Kurtyka CA, Chen L, Cress WD (2014). E2F inhibition synergizes with paclitaxel in lung cancer cell lines. PLoS One, 9, 96357.

Lee L-F, Li G, Templeton DJ, et al (1998). Paclitaxel (Taxol)induced gene expression and cell death are both mediated by the activation of c-Jun NH2-terminal kinase (JNK/SAPK). J Biological Chemistry, 273, 28253-60.

Manfredi JJ, Parness J, Horwitz SB (1982). Taxol binds to cellular microtubules. J Cell Biol, 94, 688-96.

Martinez-Cruz AB, Santos M, Lara MF, et al (2008). Spontaneous squamous cell carcinoma induced by the somatic inactivation of retinoblastoma and Trp53 tumor suppressors. Cancer Res, 68, 683-92.

Milross CG, Mason KA, Hunter NR, et al (1996). Relationship of mitotic arrest and apoptosis to antitumor effect of paclitaxel. J Natl Cancer Inst, 88, 1308-14.

Ozkaya S, Findik S, Dirican A, et al (2012). Long-term survival rates of patients with stage IIIB and IV non-small cell lung cancer treated with cisplatin plus vinorelbine or gemcitabine. Exp Ther Med, 4, 1035-8. 
RB1 Polymorphism Contributes to the Efficacy of Platinum-Taxanes in Advanced Squamous Cell Lung Cancer

Rigas JR (2004). Taxane-platinum combinations in advanced non-small cell lung cancer: A review. Oncologist, 9, 16-23.

Rozzi A, Nardoni C, Corona M, et al (2010). Weekly Regimen of Paclitaxel and Carboplatin as First-Line Chemotherapy in Elderly Patients with Stage IIIB-IV Non Small Cell Lung Cancer (NSCLC): Results of a Phase II Study. J Chemotherapy, 22, 419-23.

Schiff PB, Fant J, Horwitz SB (1979). Promotion of microtubule assembly in vitro by taxol. Nature, 277, 665-7.

Suyama H, Igishi T, Sano H, et al (2004). ERK activation and subsequent RB phosphorylation are important determinants of the sensitivity to paclitaxel in lung adenocarcinoma cells. Int J Oncol, 24, 1499-504.

van den Broek GB, Wildeman M, Rasch CR, et al (2009). Molecular markers predict outcome in squamous cell carcinoma of the head and neck after concomitant cisplatinbased chemoradiation. Int J Cancer, 124, 2643-50.

Wang MT, Zhang JJ, Xu LY, et al (2013). Invasive and prognostic significance of $\mathrm{pRB}$ in esophageal squamous cell carcinoma: a meta-analysis. Dis Esophagus, 26, 723-8.

Wang TH, Wang HS, Soong YK (2000). Paclitaxel-induced cell death: where the cell cycle and apoptosis come together. Cancer, 88, 2619-28.

Yamamoto Y, Shimizu E, Masuda N, et al (1998). RB protein status and chemosensitivity in non-small cell lung cancers. Oncol Rep, 5, 447-51.

Zhao J, Zhang Z, Liao Y, et al (2014). Mutation of the retinoblastoma tumor suppressor gene sensitizes cancers to mitotic inhibitor induced cell death. Am J Cancer Res, 4, 42-52. 